\title{
SQ-Universal 1-Relator Groups
}

\section{G. S. Sacerdote}

A countable group $G$ is SQ-universal if for each countable group $H$, there is a quotient $Q$ of $G$ in which $H$ can be embedded.

A group is a 1-relator group if it has a presentation by generators and relations involving only one relation.

Theorem: Let $G$ be a countable 1-relator group. Then either $G$ is SQuniversal or else $G$ is one of the following: (i) cyclic

(ii) metabelian and isomor-

phic to $\left.<\mathrm{a}, \mathrm{b} ; \mathrm{a}-\mathrm{l}_{\mathrm{b}} \mathrm{m}_{\mathrm{a}}=\mathrm{b}^{\mathrm{n}}\right\rangle$

where either $|m|=1$ or $|n|=1$.

Corollary: The Baumslag-Solitar group $\left\langle a, b ; a^{-1} b^{2} a=b^{3}\right\rangle$ is SQ-universal.

A group is deficient if it has a presentation in which the number of generators exceeds the number of relations by at least two. Clearly a deficient group with zero relations is SQ-universal, for it is free. By the preceding theorem a deficient group with one relation is SQ-universal. Conjecture (Peter Neumann): Every finitely generated deficient group is SQ-universal . 